\title{
Small Efforts Can Prevent Big Mistakes: Preoperative Colonoscopic Tattooing Using Indocyanine Green
}

\author{
Ji Hoon Kim, M.D., Ph.D. \\ Department of Surgery, College of Medicine, The Catholic University of Korea, Seoul, Korea
}

\begin{abstract}
The importance of tumor localization is increasing because the application of laparoscopic surgery in colon cancer surgery is on the rise and the incidence of early cancer is also rising. There are several methods of tumor localization, but the most popular method is preoperative colonoscopic tattooing. Various tattooing agents are used, and among them, India ink is the most widely used agent. However, it is impossible to use India ink in Korea. Therefore, research on other alternative agents is needed.
\end{abstract}

Keywords: Colonoscopy, Tattooing, Indocyanine green, Colorectal neoplasm

\author{
Received November 2, 2020 \\ Revised November 17, 2020 \\ Accepted December 1, 2020 \\ Corresponding author \\ Ji Hoon Kim \\ Department of Surgery, Incheon St. \\ Mary's Hospital, College of Medicine, \\ The Catholic University of Korea, 56 \\ Dongsu-ro, Bupyeong-gu, Incheon \\ 21431, Korea \\ Tel: +82-32-280-5609 \\ Fax: +82-32-280-5988 \\ E-mail: samryong@catholic.ac.kr \\ ORCID: \\ https://orcid.org/0000-0002-3093-1805
}

Copyright $\odot 2020$ The Journal of Minimally Invasive Surgery. All rights reserved. This is an Open Access article distributed under the terms of the Creative Commons Attribution Non-Commercial License (http://
creativecommons.org/licenses/by-nc/4.0/) which permits unrestricted non-commercial use, distribution, and reproduction in any medium, provided the original work is properly cited.

The need for tumor localization in colon cancer surgery is increasing. This is because the location of a tumor in colonoscopy often differs from the actual location. According to several studies, about $15 \%$ of tumor locations in colonoscopies are incorrectly identified during operations. ${ }^{1-3}$ Therefore, it is important to accurately locate the tumor during surgery. This can prevent the catastrophe of resecting another segment of the colon. There are two reasons why it is difficult to accurately locate a tumor during surgery. The first is that laparoscopic surgery is widely used in colon cancer surgery, but it has less tactile sensation than open surgery. The second is that the tumor is small due to the increase in early cancer detection and rise in surgeries after endoscopic mucosal resection or submucosal dissection.

There are three methods to determine the location of a tumor during surgery. The first one is to perform colonoscopy dur- ing surgery. However, this increases the operation time, and the distension of the intestine can make the operation difficult. The second method is to use a metal clip under colonoscopy before surgery. This has the disadvantage that there may be cases where the lesion cannot be found due to loss of the clip. ${ }^{4}$ The third method is colonoscopic tattooing before surgery. However, there are still few comparative studies on which of these three methods is the best.

There are several colonoscopic tattooing agents. Autologous blood does not cause bowel inflammation and complications from spillage that may occur with other tattooing agents. However, additional blood collection is required, and the accurate location may be difficult to find due to bleeding during surgery. India ink is the most common tattooing agent. ${ }^{5}$ Therefore, it is widely used worldwide, but is not currently available in Korea. In 
this current situation, this study is meaningful because it proves the usefulness of indocyanine green (ICG) as a tattooing agent with a comparative study between India ink and ICG.

In this study, it was proved that there was no difference in the visualization rates of the India ink group and the ICG group. In addition, the advantage of this paper is the analysis of open surgery and laparoscopy surgery, which may have a bias caused by tactical sensation.

However, this is a retrospective study and has some limitations. First, the dye leakage rate was not analyzed. This is because the complications of most tattooing agents are the result of dye leakage. The advantage that ICG has over India ink is that unlike India ink, it is a liquid component, so there is little contamination in the operation field in case of dye leakage. Another advantage of ICG is that it can be detected using fluorescence, while tattooing is difficult to find. However, this study could not analyze this.

Even so, the strength of this study is that if tattooing is performed only a few days before surgery (due to the short durability of ICG), ICG can be an excellent alternative to India ink.

\section{ORCID}

Ji Hoon Kim, https://orcid.org/0000-0002-3093-1805

\section{CONFLICT OF INTEREST}

None.

\section{FUNDING}

None.

\section{ACKNOWLEDGMENTS}

None.

\section{REFERENCES}

1) Cho YB, Lee WY, Yun HR, Lee WS, Yun SH, Chun HK. Tumor localization for laparoscopic colorectal surgery. World J Surg 2007;31: 1491-1495.

2) Vignati P, Welch JP, Cohen JL. Endoscopic localization of colon cancers. Surg Endosc 1994;8:1085-1087.

3) Acuna SA, Elmi M, Shah PS, Coburn NG, Quereshy FA. Preoperative localization of colorectal cancer: a systematic review and metaanalysis. Surg Endosc 2017;31:2366-2379.

4) Kim SH, Milsom JW, Church JM, et al. Perioperative tumor localization for laparoscopic colorectal surgery. Surg Endosc 1997;11:10131016.

5) ASGE Technology Committee, Kethu SR, Banerjee S, et al. Endoscopic tattooing. Gastrointest Endosc 2010;72:681-685. 